\title{
Thank you
}

The guest editors would like to thank those listed below for constructive and insightful reviews of the manuscripts. Your expertise has contributed importantly to this special issue.

Bjørn Hofmann, University of Oslo/Gjøvik University College, Norway

Ellen Wright Clayton, Vanderbuilt Law School, USA

Fay Betsou, Integrated Biobank of Luxembourg, Luxembourg

Georges Dahger, INSERM, France

Håkon Gjessing, Norwegian Institute of Public Health, Norway

Jaakko Kaprio, University of Helsinki, Finland

Juha Muilu, University of Helsinki, Finland

Julian Little, University of Ottawa, Canada

Kirsten Ohm Kyvik, University of Southern Denmark, Denmark

Kristian Hveem, Norwegian University of Science and Technology, Norway

Kristin Andersson, Lund University, Sweden

Martin Yuille, University of Manchester / Manchester Academic Health Science Centre, UK

Nadja Kanellopoulou, HeLEX, UK

Ruth Chadwick, Cardiff University, UK

Stefan Eriksson, Uppsala University, Sweden 